\title{
AMOR A LA DIFERENCIA/AMOR EN LA DIFERENCIA. REFLEXIONES ACERCA DEL 'ESPACIO DEL SILENCIO' \\ EN LA OBRA DE LUCE IRIGARAY
}

LOVE FOR DIFFERENCE.

NOTES ON SILENCE IN LUCE IRIGARAY

\author{
Virginia Fusco \\ Universidad Carlos III de Madrid
}

\section{RESUMEN}

Desde los años noventa venimos asistiendo a un creciente interés por lo que Ann Ferguson ha definido como el neonato campo de los Love Studies (2017). El análisis acerca de las prácticas y los discursos amorosos se ha ido articulando, progresivamente, alrededor de reflexiones que pretenden dar respuesta a qué es el amor y/o cómo opera en tanto que ideología, epistemología y fuerza psicosocial. Estas tres maneras de entender el fenómeno del amor, más allá de sus representaciones en la cultura contemporánea, revelan la compleja genealogía de las formas en que la noción de amor se ha introducido en el análisis de las relaciones que vinculan a hombres y mujeres, en concreto, en relaciones heterosexuales, así como en la posible construcción de una ética/erótica hetero, fundada en el reconocimiento mutuo. En este acercamiento a la situación anteriormente descrita me propongo indagar sobre el rol que ocupa el amor en (el feminismo de) la diferencia o, como sostiene Irigaray, acerca del rol que el amor a la diferencia desempeña en la creación de un orden social y simbólico que no excluya lo femenino. En particular, analizaré un conjunto de obras (Amo a ti: bosquejo de una felicidad en la historia, Ser dos y Ética de la diferencia sexual) en las que la autora francesa explicita cómo el paso de una cultura de lo Mismo a una cultura del Dos, es decir, a una cultura que privilegia el amor al otro y a lo otro, se vuelve posible gracias a relaciones intersubjetivas, fundadas en la práctica del silencio. Además, con esta breve intervención, pretendo abordar un aspecto muy relevante de la obra de Luce Irigaray que no ha sido suficientemente estudiado y que podría contribuir a reinterpretar el feminismo de la diferencia, atendiendo a lo relacional como elemento fundacional para la construcción de la subjetividad femenina.

Palabras clave: Love Studies, Irigaray, feminismo de la diferencia, el espacio del silencio, relacionalidad. 
ABSTRACT

In 2017, Ann Ferguson remarks that from the nineties there has been a growing interest in Love and that Love Studies has become a new field of feminist enquiry.

The analysis of a number of discourses on love and love practices has been articulated in the attempt to answer the question of what love is and how it operates as an ideology, an epistemology and a psychsocial force.

These three modes of interpretation that move beyond the analysis of the forms in which love is culturally apprehended and represented, reveal the complex genealogy of the strategies through which the notion of love has been used to understand relationships between men and women and as a key element to possibly construct an heterosexual ethics/erotica funded on mutual recognition.

In this paper, I will focus on the role that Love plays in (feminism of) sexual difference or, as Irigaray states, on the role that Love for Difference performs in the creation of a social and symbolic order that does not exclude the feminine. In particular, I will analyse a body of work (I Love to You: Sketch for a Felicity Within History, To Be Two and An Ethics of Sexual Difference) in which the author explains how Love would operate to remodel the culture of the Same into a culture of Two, a culture that will be made possible by inter-subjective relations founded on the practice of silence. Moreover, I wish to consider a very relevant aspect of Irigaray's theorising that has not been sufficiently accounted for and that would allow to re-think the role of relationality in constructing female subjectivity in Feminism of the Sexual Difference.

Keywords: Love Studies, Luce Irigaray, Feminism of the Sexual Difference, Silence, Relationality.

\section{SUMARIO}

1.- Introducción. 2.- Cronología de un proceso. 3.- La herencia imposible. 4.- Herederos del acontecimiento. 5.- El porvenir del pasado. 6.- Bibliografía

\section{Love Studies: hacia una cartografía}

Ann Ferguson y Margaret Toye, en la extensa introducción al volumen monográfico sobre el amor publicado por la revista Hypatia en 2017, ponen el acento en el creciente interés que, a partir de los años noventa, un número relevante de investigadoras demuestran por un campo de investigación que hasta entonces había ocupado un lugar marginal y subalterno. La reflexión acerca del rol que el amor desempaña en la construcción de las relaciones íntimas y, quizás, de muchas de nuestras relaciones interpersonales, se entendía hasta este momento como expresión de un 'saber sometido', o sea, como sostiene Foucault, formas de 
conocimientos que «estaban descalificados como saberes no conceptuales, como saberes insuficientemente elaborados: saberes ingenuos, saberes jerárquicamente inferiores, saberes por debajo del nivel del conocimiento o de la cientificidad exigidos» (Foucault, 2001: 21). El monográfico de Hypatia recoge reflexiones propuestas por académicas de diversos campos disciplinares y variada formación que ya no se limitan a analizar las representaciones del amor que encontramos en el arte, la literatura o en la producción audiovisual contemporánea sino que pretenden reflexionar sobre el amor en its own terms. Las autoras revelan la importancia de la crítica feminista en la definición de este nuevo campo cuando remarcan cómo el movimiento de las mujeres desde sus comienzos y sus vanguardias teóricas han elaborado formas de entender el amor como emoción que promueve y consolida las relaciones de subordinación de las mujeres en la sociedad patriarcal (Esteban, 2011 : 39-42).

En un volumen más antiguo, Love. A question for Feminism in the Twenty-First Century (2015), que formalmente inaugura, dentro el contexto académico, la reflexión sistemática acerca del amor y en el que se definen los términos claves que nos orientan en la ya amplia producción de los Love Studies, Ann Ferguson y Anna Jónasdóttir identifican básicamente tres perspectivas interpretativas que articulan las miradas de las investigadoras feministas sobre el fenómeno: Love as delusion/ideology, Love as a key element in epistemology y Love as social human power. Dicho con otras palabras, desde estas perspectivas interpretativas se pondría de manifiesto una crítica al amor como ideología al servicio del patriarcado, una versión del amor entendido como un poder emotivo irreprimible que se expresa en la creación de una episteme relacional humana $y$, por último, el amor como energía que se manifiesta en un conjunto complejo de prácticas individuales y colectivas y no simplemente en una dimensión puramente discursiva.

Una lectura atenta de las distintas contribuciones que el segundo volumen aglutina revela que estos bloques están caracterizados por una enorme heterogeneidad interna; entiendo que los distintos contextos de producción -la academia así como ámbitos vinculados a la militancia feminista- determinan en buena medida esta diversidad de propuestas. En todo caso, me parece importante subrayar que estos mismos contextos diferenciados imponen la necesidad de trazar y reconstruir genealogías precisas para poder dar cuenta de las formas en las que la noción de amor ha sido útil para pensar las relaciones entre los sexos y, en particular, las prácticas amorosas que vinculan íntimamente a hombres y mujeres (Singer, 2009). En efecto, históricamente, gran parte de la crítica feminista europea y norteamericana se ha dedicado al análisis del amor romántico o sea, de una forma de amor que en la edad de la hegemonía de la burguesía como clase se expresa y consolida en relaciones heterosexuales sancionadas por el matrimonio. A este tipo de ideología amorosa-que se 
manifiesta, especialmente entre finales del siglo diecinueve y mediados del siglo veinte, como fuerza destructora y como poder coercitivo- se refiere la vasta mayoría de las elaboraciones teóricas feministas más antiguas, desde Mary Wollstonecraft (1996), pasando por la obra de Harriet Tylor y Stuart Mill (1970), hasta las más recientes contribuciones de Shulamith Firestone (1971) y Monique Wittig (1992).

Es justo a partir de estas consideraciones que algunas otras teóricas de los años setenta del siglo XX -cabe aquí recordar la vehemencia de Adrienne Rich (1980)-abrazan teóricamente la fuga de un vínculo jurídico y social ideológicamente fundamentado en el amor romántico como arma preferencial para articular otras prácticas amorosas fuera de la mente hetero y contra ella. Para estas autoras habrá que edificar otras formas de amor entre sujetos que nos liberen como mujeres de la desigualdad y nos permitan fundamentar prácticas relacionales que favorezcan la emancipación. Entre las varias propuestas se registra un énfasis creciente, principalmente en el contexto norteamericano, en el amor y la erótica lésbica como respuesta a la hegemonía de la mente hetero y como punto de fuga a las relaciones de opresión (Whelehan, 1995).

A partir de los tres ejes que aglutinan la crítica feminista acerca del amor -identificados, como decía, por Ferguson y Jónasdóttir- me propongo analizar la peculiar conceptualización del amor que nos ofrece Luce Irigaray, filosofa y psicoanalista francesa. Analizaré un conjunto de textos en el intento de iluminar cómo su concepción del amor como epistemología representa, desde mi punto de vista, un terreno muy fecundo para repensar el amor desde el feminismo y, a la vez, ofrecer una perspectiva sobre las relaciones amorosas que no excluya implícita o explícitamente cualquier expresión de una erótica heterosexual entendida necesariamente, desde la actual persistencia del modelo romántico, como expresión de relaciones de subyugación.

Pues, en la obra de Irigaray, el amor, entendido como epistemología, resulta indispensable para promover una revolución de lo cotidiano que forjará no solo un nuevo mundo fundado en el reconocimiento de los 'derechos' de las mujeres sino que permitirá la construcción de una red de relaciones simbólicas en las que se desplazará el dominio de lo Mismo en pro de un mundo de Dos, en el que se podrá desplegar y expresar una subjetividad femenina emergente.

\section{Luce Irigaray y la diferencia sexual} en la modalidad del todavía no (del ser). 
En la reflexión de Irigaray que pivota alrededor de las relaciones entre los sexos y de los procesos que permiten el despliegue de la subjetividad femenina en un universo dominado por lo Mismo, el amor juega un rol esencial en la construcción de un orden simbólico y social que no excluya lo femenino. En este sentido las intuiciones de la teórica francesa acerca del amor como sentimiento y pasión, que claramente tiene una función política en la construcción de un mundo nuevo, se enmarcan en lo que las teóricas de los Love Studies han identificado como epistemología y a la vez se desbordan en el campo del amor entendido como un conjunto de prácticas individuales y colectivas de los cuerpos sexuados.

Para entender la forma en la que Irigaray conceptualiza el amor como epistemología emancipadora en primer lugar es indispensable recordar que para la teórica francesa la diferencia sexual, en la cultura falocrática o falocéntrica (Wright, 1992: 316 ) en la que vivimos, no existe como un a priori. Irigaray señala como, en sociedades patriarcales, el discurso está organizado alrededor de la idea de masculinidad que tácitamente funciona como género normativo. En su elaboración el falo opera como un significante privilegiado, o sea, funciona como garantía ideal de la articulación del significado dentro de un sistema de conocimiento mediado y organizado a través del lenguaje. Es pertinente aquí recordar la definición que Lacan (psicoanalista de referencia para Irigaray y para la crítica postestructuralista) hace del falo:

El falo no es una fantasía, si hay que entender por ello un efecto imaginario. No es tampoco como tal un objeto (parcial, interno, bueno, malo, etc...) en tanto que ese término tiende a apreciar la realidad interesada en una relación. Menos aún es el órgano, pene o clítoris, que simboliza [...] Pues el falo es un significante [...] Pues es el significante destinado a designar en su conjunto los efectos de significado, en tanto que el significante los condiciona por su presencia de significante. (Lacan, 1987: 381).

Real, Simbólico, Imaginario constituyen en Lacan una triada de conceptos cuyo uso está totalmente alejado del empleo que de estas palabras se hace en el lenguaje ordinario y, por esta razón, resulta difícil acotar el significado del falo en la economía discursiva psicoanalítica posestructuralista a la que la obra de Irigaray se adscribe (Benvenuto y Lucci, 2014). No obstante, hay acuerdo en sostener que el falo como significante simbólico se constituye como un elemento que garantiza cierta estabilidad del significado y que opera en un registro alejado de la experiencia. A la vez, el falo parece colapsarse en la realidad fenomenológica y resulta ambiguamente encarnado de forma privilegiada en los sujetos masculinos (Irigaray, 1985: 28).

Para Irigaray, entonces, a partir de estas nociones elaboradas en el seno del psicoanálisis freudiano y posteriormente por Lacan, la preeminencia atribuida a un sexo, 
el masculino, en la economía simbólica y la supresión, en este orden, vacía toda posible identificación de una feminidad en efecto ausente.

En lugar de primar consideraciones acerca del poder patriarcal y las estructuras económicas e institucionales de opresión, el análisis de Irigaray se articula sobre los efectos discursivos de la subordinación femenina y analiza cómo el dominio de lo Mismo construye la posición simbólica del sujeto mujer desde una dialéctica negativa como 'falta de' y simplemente como espejo - hecho de cuerpo y materia- que permite al sujeto masculino su diferenciación y constitución. Así pues, desde esta perspectiva, las mismas condiciones de subjetivación imposibilitan hablar de una feminidad en cuanto tal, como nos recuerda Dylan Evans:

There is no signifier of sexual difference in the symbolic order. The only sexual signifier is the phallus, and there is no 'female' equivalent of this signifier: 'strictly speaking there is no symbolization of woman's sex as such...the phallus is a symbol to which there is no correspondent, no equivalent. It's a matter of a dissymmetry in the signifier' (Evans, 1996: 182).

Lo femenino - de aquí la propuesta de una lectura no esencialista de la diferencia cómo expresión de un devenir-mujer en línea con la elaboración de Braidotti en Metamorfosis-, tiene todavía que emerger y constituirse; una subjetividad en construcción que no es complementaria y especular al hombre sino una encarnación compleja y constituida por múltiples capas alejada de la institución de la feminidad. (Braidotti, 2002: 11)

El lenguaje y los sistemas de representación en este universo discursivo falogocéntrico no pueden dar cuenta -traducir dirá la psicoanalista francesa (Irigaray, 1977)- del deseo femenino ni la experiencia de ser mujer en el mundo. En efecto, el sistema de parole, discurso y lógica se revela no neutral y elemento esencial para que se construya y consolide, en el tiempo, la supremacía de lo masculino.

En una entrevista del 2013, Irigaray afirma:

I am afraid that women have not yet understood what sexuate difference means and what, not only natural but also cultural, resources lie in relationships in difference. They remain divided between a not-yet-cultivated feminine part of themselves and a culture in the masculine that they still see as the only possible culture that they must reach. (Irigaray, 2013: 2). La cursiva es mía.

La condición de posibilidad del devenir-mujer -o sea de que la diferencia sexual represente simultáneamente un hecho anatómico y fisiológico, un estado psicosocial y una posición subjetiva- «representa una de las cuestiones o la cuestión que debe pensarse en 
nuestra época» porque "la diferencia sexual podría constituir el horizonte de mundos de una fecundidad todavía no advenida» (Irigaray, 2010: 35):

Es verdad que, para que tenga lugar la obra de la diferencia sexual, se precisa una revolución de pensamiento, y de ética. Todo tiene que ser reinterpretado en las relaciones entre el sujeto y el discurso, el sujeto y el mundo, el sujeto y lo cósmico, el micro - y el macrocosmos (Irigaray, 2010: 36).

En este proceso revolucionario de reinvención de lo que podríamos definir como el 'conjunto' de nuestro mundo, y en oposición a una cultura donde lo masculino se ha construido y cultivado como lo neutro -lo no generizado/sexuado y lo universal-, habrá que contraponer 'una cultura de Dos' entendida como cultura fundada en el encuentro que genera asombro, maravilla y admiración frente a lo (él/ella) que es inaprensible en cuanto otro radical (Irigaray, 2010: 42) y que, no obstante, resulta reconocible. Este encuentro se da en el espacio relacional del amor teorizado por la psicoanalista francesa como el espacio del silencio.

\section{El silencio y el amor}

From speculum on, my project has been how to render possible a philosophy, and more generally, a culture of two subjects

En el universo falocéntrico prima lo Mismo y los dos sexos autónomos colapsan en un modelo de humanidad singular y universal donde la diferencia de dos cuerpos anatómicamente diferenciados se borra en pro de un sujeto neutral ficticio y su doble negativo. El paso de una cultura del Uno y su negativo a una cultura del Dos, o sea, a una cultura de la multiplicidad y de la pluralidad, es fundacional de un orden donde lo femenino se revele como diferencia no dialéctica.

En la perspectiva aquí ilustrada, el lenguaje juega un rol esencial en la definición de esta neonata subjetividad femenina; un lenguaje poético que crea, que conserva sus cualidades sensibles y así se dirige al cuerpo y al alma y se vuelve viviente (Irigaray, 2008: 14). En este sentido, la teórica francesa explora, en el conjunto de su obra más tardía, las maneras de elaborar nuevas formas de comunicación intersubjetiva (lingüística y no lingüística) para la creación de esta cultura del Dos. 
En Amo a ti, Irigaray indaga -como bien se indica en el título - la posible transformación de relaciones objetuales con los otros en relaciones intersubjetivas de reconocimiento. Si atendemos a la construcción gramatical de la forma en la que habitualmente decimos te amo, observamos como el 'te' se construye lingǘsticamente como el objeto de mi propio deseo; el verbo indica el sujeto que ama y desea y apunta hacia un 'objeto' receptor pasivo, lugar inmóvil donde se proyectan mis fantasías de encuentro imposible. Amo a ti opera como deconstrucción de la relación objetualizada en pro de una relación intersubjetiva y dialógica de reconocimiento mutuo.

Irigaray así lo expresa en una entrevista:

\begin{abstract}
"Amo a ti" significa: no te tomo como objeto de mi amor ni de mi deseo. Te quiero como irreductiblemente otro. Mantengo la " $a$ " como un espacio inalienable entre nosotros, garantía de tu libertad y de la mía. "Amo a ti" significa que conservo contigo una relación de indirección para evitar toda posesión o consumación amorosa y proteger de este modo el dos que somos [...]."Amo a ti" significa que nunca te conoceré totalmente y que amarte implica respetar el misterio que tú siempre serás para mí. (Irigaray, 1994: 178)
\end{abstract}

Para Irigaray, el te amo revela las formas en las que las relaciones interpersonales se pueden adscribir a la lógica de lo Mismo; el otro no tiene estatuto de sujeto ni goza de la posibilidad de ser reconocido, sino que constantemente es objetivado y construido como lienzo proyectivo sobre el que se despliegan las fantasías narcisistas de uno mismo. No hay encuentro; el otro es silenciado, asimilado y, en última estancia, destruido como autonomía. Lo que se consume en las relaciones amorosas tal como se forjan en la cultura falocéntrica es un amor solipsista autorreferencial.

Ya Annie Leclerq y Hélène Cixous, pensando en el dominio de lo Mismo, habían remarcado la necesidad del cultivo de una voz propia, de un hablar en primera persona y en femenino y así construir un lugar de resistencia a la hegemonía de lo masculino.

Leclerc sintetiza perfectamente aquí la necesidad de un habla femenina:

Nada existe que no sea hecho por el hombre, ni el pensamiento, ni el lenguaje, ni la palabra. Nada existe aún que no sea hecho por el hombre; ni siquiera yo. Sobre todo yo. Todo está por inventarse. Las cosas del hombre no sólo son estúpidas, mentirosas y opresivas. Son sobre todo tristes, tristes hasta morir de aburrimiento y desesperación. Inventar un lenguaje de mujer. Pero no de la mujer como es dicha en el lenguaje del hombre; porque este lenguaje es fastidioso, repite siempre lo mismo (Leclerc, 1977: 6). La cursiva es mía 
En este sentido es indispensable la búsqueda de una voz propia y, también, de una escritura femenina y en femenino para desarmar el universo singular del falo; este desarme es la precondición de un encuentro, de una abertura a la posibilidad del amor.

De manera quizás anti-intuitiva y contradictoria Irigaray sostiene que la búsqueda de voz está íntimamente ligada al espacio de silencio. En efecto, en lugar de enfatizar solamente la necesidad de romper un silencio histórico que ha rodeado a las mujeres durante siglos y renegar de la práctica histórica de la escucha -entendida como 'cuidado'- de un emisor masculino privilegiado, Irigaray elabora una personal visión del silencio que concibe como actitud y práctica y, simultáneamente, como espacio que posibilita el sustraerse al dominio del falo para así construir un universo plural y múltiple donde el amor se vuelva posible.

El punto de departida de la reflexión es que el silencio se presenta como un hecho esencial en la comunicación humana. De forma intuitiva podemos reconocer que el silencio de aquel que escucha posibilita el habla. Si históricamente hemos pensado el silencio como expresión de la pasividad femenina en las relaciones de poder entre los sexos, también podemos entenderlo como una actitud y una práctica que se encuentran íntimamente vinculadas a la escucha. La oposición entre actividad y pasividad -uno de los binomios sobre los que se ha gestado la jerarquía simbólica entre los sexos y la irrelevancia de lo femenino como no-sujeto en cuanto que pasividad- ya no tienen lugar aquí.

Te escucho tal como otro que me trasciende, requiere el pasaje a una nueva dimensión. Te escucho: percibo lo que dices, estoy atento(a) a ello, intento captar tu intención. Esto no significa: te comprendo, te conozco, por tanto no tengo necesidad de escucharte... te escucho facilita lo todavía no-codificado, el silencio, un sitio de existencia, de iniciativa, de intencionalidad libre, de sostén a tu devenir (Irigaray, 1994: 167).

El silencio y la escucha, entendidos como interdependientes y mutua condición de posibilidad, acontecen como elementos no-pasivos en el espacio de la relación. Es en este espacio, que se construye a través del reconocimiento y que adquiere una dimensión temporal en su devenir, donde el amor puede darse como hecho al servicio de la diferencia sexual para que esta misma pueda emerger en la cultura falocéntrica.

Entonces debemos buscar la palabra para decir, pero también el silencio. Al conectar el mundo gracias a una sola palabra, el otro no se puede decir y hablar con él se ha vuelto imposible. Porque hay mundos diferentes, que requieren silencio para decir, escuchar, comunicarse. En este sentido, encontrar los términos correctos es un obrar in-finito [...].(Irigaray, 2008: 17) Traducción mía. 
Así, cuando se guarda silencio se brinda al otro la posibilidad de hablar y de transformarse en sujeto a través de una reapropiación, reinscripción de un lenguaje que emana de cuerpos que han sido conformados de forma distinta. Dicho con otras palabras, el silencio no se presenta como el marco relacional en el que un 'objeto' (las mujeres) es forzado a la escucha de un otro hegemónico en la cultura patriarcal de lo Mismo; sino que el silencio como práctica permite que un objeto sea transfigurado en sujeto (Amo a ti) gracias a la acción de escuchar conscientemente, desde un ejercicio de la voluntad de estar en comunión y de comunicar(se) con un sujeto de amor que ya no es mío, absorbido por mi deseo y poseído desde la lógica del no reconocimiento.

Siguiendo esta lectura, este silencio es entonces el lugar en el que acontece una apertura al otro:

En lugar de llevar al otro a una casa de lenguaje ya establecida en sus fundamentos, el sujeto se arrastra ante la irreductibilidad del otro. Hacer silencio: un silencio que no es el que ya está impreso en el despliegue de la palabra, un silencio que suspende este despliegue para abrir el espacio de otro despliegue. (Irigaray, 2008: 30) Traducción mía

A partir de estas reflexiones fragmentarias sobre el espacio del silencio en la construcción de un mundo de Dos, Irigaray sostiene que el silencio ha de ser cultivado y protegido como acontecimiento 'precioso' en el camino del reconocimiento de la diferencia. Aquí también hay un juego de múltiples significaciones del término precioso; precioso en cuanto extremadamente valioso y, a la vez, precioso como adjetivo que se refiere a la admiración -la pasión de la diferencia (Irigaray, 2010: 42)- por su belleza como momento fundacional de un orden simbólico dual.

Tal como decía anteriormente, la noción de silencio en la escritura de Irigaray adquiere también otro matiz cuando habla en Essere due (1994) de este lugar del silencio que no existe solamente como realidad externa al sujeto en la comunicación con el otro, sino más bien como el despliegue de un espacio interno al mismo sujeto y que en la relación intersubjetiva de los amantes siempre acontece como un dos:

Compartido no de forma sencilla: debo salvaguardar el silencio en mí, y debo respetar el silencio del otro. De esta manera, el silencio son dos: dos irreductibles entre sí, dos irreductibles a uno. (Irigaray, 1994: 73) Traducción mía

En otros términos, el momento comunicativo para Irigaray se construye triangularmente: mi propio silencio, el silencio del otro y el silencio que posibilita el espacio relacional. El 
silencio representa una etapa del devenir-hombre y del devenir-mujer así como un elemento imprescindible de la relación; un silencio que siempre es tres. Este espacio no se construye discursivamente como propiedad de uno o de otra en la relación sino que es generado, como creatura en el lugar fecundo del silencio, entre los dos y por lo tanto responde a su propia lógica fuera de lo Mismo y de su dominio. Irigaray retrata este espacio de silencio relacional como no asimilable, un lugar en el que no podemos superar al otro, ni la relación con él/ella a través de su objetivación.

En última instancia, Irigaray conceptualiza la experiencia del silencio (práctica y espacio) como comunicación y comunión entre dos singularidades que se despliegan y no son reducidas a una unidad. Si ponemos en relación sus intuiciones acerca del amor con la lógica cultural del amor romántico, este último se revela como expresión de la voluntad de subsunción de uno de los dos elementos constitutivos de la relación amorosa, el femenino, y su asimilación en un uno indiferenciado donde la diferencia solo se puede concebir como complementariedad o servicio.

A diferencia del sistema de parole, discurso y lógica dominado por lo Mismo, que aniquila la posibilidad del habla femenina y que, en la cultura falogocéntrica, niega la escucha como acción, la práctica del silencio y el espacio que desde esta se genera remarca la posibilidad de la construcción de una ética amorosa de la diferencia sexual que tenga en cuenta la singularidad de cada uno.

Consecuentemente con ello, la única postura ética sostenible -en el caso de la comunicación/comunión entre sujetos- sería la que emana de una nueva cultura del silencio que, por un lado, resiste la tendencia del lenguaje a producir asimilación, exclusión y jerarquías $y$, por otro, posibilita el emerger del Dos, o sea la valorización de la diferencia sexual como diferencia primigenia no-jerarquizada.

La posibilidad de hacer(se) oír, de hablar, de representar y de encontrar una voz propia se articula como resignificación y feminización de un universo discursivo androcéntrico; el silencio vehicula y moviliza la potencialidad de una comunicación nueva que omite los sistemas binarios, jerárquicos y excluyentes. Irigaray pretende solventar los límites de una reflexión filosófica acerca de la palabra como nombramiento y colocar en el centro el silencio como expresión de una nueva episteme amorosa.

Esta reflexión conceptual acerca del amor como epistemología y como conjunto de prácticas se asemeja, a primera vista, a una reflexión acerca del espacio de la comunicación-no-verbal. En Irigaray la necesidad de revisión del lenguaje masculino, condición de posibilidad de una lengua femenina, implica su total subversión: desde la re-significación de la palabra pronunciada hasta la exploración del potencial de lo no dicho, de lo que se 
calla. Por otra parte, el vínculo íntimo que la autora establece entre amor y silencio parece apuntar a una noción de lo no-verbal como gesto entendido como aquello que se expresa simplemente a través del cuerpo sin necesidad de mediación lingüística; ya Merleau-Ponty, en su Fenomenología de la percepción, reconocía en los gestos la posibilidad de expresar una nueva forma de ser y revelar nuevas maneras de habitar el mundo y de hacer mundos (en Taylor, 2016: 29).

La apología del silencio en Irigaray se revela vinculada a una particular forma de entender lo no-discursivo y su nexo con el orden simbólico, especialmente si tenemos en cuenta que la propia practica psicoanalítica descansa sobre un espacio relacional que cultiva el silencio y la escucha. Cabe preguntarse si la interpretación que la autora francesa ofrece de lo no-discursivo corresponde al lugar y la temporalidad donde lo femenino no está subsumido todavía por el falo. Algunas teóricas han hecho coincidir lo no-discursivo en Irigaray con la fase pre-edípica de la no diferenciación del feto de la madre y, en este sentido, han hablado de una problemática dimensión regresiva en su aproximación:

That time before femeninity or masculinity when the infant is in symbiotic relation with the mother. [...] for Irigaray [...] it is the point at which femininity has not yet been repressed by patriarchy and women have not yet become manmade. (Weedon, 1987: 56)

En esta línea, la apuesta por una dimensión no-discursiva coincidiría con la exclusión de las mujeres del lenguaje y, por consiguiente, de lo simbólico. Peligrosamente se reforzaría una idea de feminidad totalmente alejada de lo humano entendido como animal lingǘstico; en última instancia, si aceptamos esta interpretación, parece implícita una crítica a la noción de silencio en Irigaray, como concepto que, en el plano teórico, refuerza una concepción misógina de una feminidad apartada de la cultura, entendida como pura inmanencia.

Sin embargo, me parece que lo que pretende decir aquí Irigaray es que el silencio se constituye como espacio de negación voluntario del discurso falogocéntrico, o sea, como posibilidad de los sujetos encarnados de sustraerse a la parole, al discurso y a la lógica de lo Mismo. El silencio no sería pensable en relación con la regresión y la aniquilación de toda posibilidad de individualización, sino como posibilidad de resistencia a través de una renuncia. Por otra parte, este espacio del silencio hay que entenderlo como constantemente en construcción y deconstrucción y en un devenir; no representa un lugar en un sentido topográfico ni tampoco hay que confundirlo con una etapa evolutiva de la configuración psicosocial del sujeto; no tiene un vínculo estrecho con una condición o estadio que tenemos que alcanzar o al que tenemos que volver en una fantasía narcisista de recuperar el silencio del vientre materno. Considero que, justo por esta razón, los escritos de Irigaray no reflejan nin- 
guna dimensión normativa en su discurso acerca del silencio, ninguna práctica concreta que deba ser añadida a su apelación a la escucha y al reconocimiento del otro como unicidad irreducible. La vindicación del silencio es un gesto con una capacidad de crear un mundo que todavía no se ha realizado y/o que no se realizará nunca plenamente.

\section{Amor a la diferencia/amor en la diferencia}

Lo que he pretendido mostrar aquí es la forma en la que la reflexión de Irigaray acerca del Amor y el rol que el silencio desempeña en ella contribuye de forma novedosa a la investigación llevada a cabo en el marco de los Love Studies. Entre los distintos modos de pensar el amor que han sido elaborados a partir de los años noventa del siglo pasado, la noción de amor que se despliega en la obra de la teórica francesa nos dirige hacia una epistemología situada en la experiencia de la diferencia y a su capacidad de crear un espacio de relación entre sujetos sexuados no organizados a través de la economía simbólica de lo Mismo. Alejándose de un análisis de las formas en las que la cultura contemporánea reiteradamente construye representaciones del amor en gran parte articuladas por la subordinación de lo femenino, la autora promueve, en el conjunto de su obra, una nueva visión del amor como lugar privilegiado de acceso a una economía relacional que posibilita el emerger de un mundo del Dos, o sea de un devenir de la diferencia cuya expresión primigenia se encarna en la diferencia sexual.

Dicho de otro modo, el amor a la diferencia y en la diferencia constituye el pivote que permite la creación de un orden social y simbólico que no excluya lo femenino, un orden que desplaza el falogocentrismo en pro de relaciones intersubjetivas de reconocimiento y de individuación. Una de las estrategias que permite este desplazamiento del falo y del logos, esta salida de un amor solipsista y autoreferencial, está representada por la práctica del silencio. Lo que he pretendido mostrar es que el silencio, aunque se configure como dispositivo que conduce simultáneamente a una práctica y a un espacio, posibilita el encuentro de dos autonomías que se sitúan fuera de lo discursivo, fuera del dominio lingüístico de lo Mismo. En este escenario, el silencio posibilita el Amor a la diferencia, al otro, como hecho al servicio de la diferencia sexual. En última instancia, Irigaray sostiene que el dispositivo del silencio promueve el emerger de una cultura de la multiplicidad y de la pluralidad donde el Otro y lo otro se revelan como diferencias no dialécticas. Así pues, la apología del silencio en Irigaray se revela vinculada a una particular forma de entender lo no-discursivo y su nexo con el orden simbólico. Si atendemos a la reflexión coherente que la psicoanalista francesa disemina en este conjunto de textos que he analizado, el silencio se constituye como espacio 
de posibilidad para los sujetos encarnados de sustraerse a la lógica de lo Mismo a través de la renuncia y de una resistencia que pasa por la negación de la palabra y de su lógica binaria de exclusión/inclusión. Si, como nos advierte Irigaray, la diferencia sexual emerge en el espacio del silencio que se sustrae a la economía de lo Mismo, no se podría hablar entonces de una diferencia ya constituida, sino de una diferencia no dada ontológicamente sino entendida como abertura al espacio de la relación en un devenir temporal nunca completamente agotado.

La reflexión acerca del silencio y de la posibilidad de construir un universo del Dos pretende solventar un vacío que se detecta en la reflexión filosófica occidental y en la filosofía feminista en particular.

\section{Bibliografía}

BRAIDOTTI, Rosi (2002). Metamorphoses. Towards a materialist theory of becoming, Cambridge: Polity Press.

Esteban, Mariluz (2011). Critica del pensamiento amoroso. Temas Contemporáneos, Barcelona: Edicions Bellaterra.

Ferguson, Ann and Anna G. JónASdótTiR (eds.) (2015). Love. A Question for Feminism in the Twenty-First Century, London: Routledge.

Ferguson, Ann and Margaret Toye (eds) (2017). "Feminist Love Studies. Editors' Introduction". En línea: https://onlinelibrary.wiley.com/doi/abs/10.1111/hypa.12311. [Consulta: 11.09.18].

Firestone, Shulamith (1971). The Dialectic of Sex: the Case for Feminist Revolution, New York: Bantam Book.

Foucault, Michel (2001). Defender la sociedad. Curso en el Collège de France (1975-1976), Méjico: Fondo de Cultura Económica.

IrIGARAY, Luce (1985). Speculum of the Other Woman, New York: Cornell University Press.

- (1994). Essere due, Torino: Bollati Boringhieri.

- (1994). Amo a ti. Bosquejo de una felicidad en la historia, Barcelona: Icaria.

- (2008). La via dell'amore, Torino: Bollati Boringhieri.

JÖNASDOtTIR, Anna G. (2015). «Love Studies. A (Re)New(ed) Field of Konwledge Interests» en FERGUSON, Ann and Anna G. JóNASDÓtTIR (eds) (2015). Love. A Question for 40

Feminism in the Twenty-First Century, London: Routledge, pp. 11-30. 
LACAN, Jaques (1987) «La significación del falo», en Jaques Lacan, Escritos II, Buenos Aires: Siglo Veintiuno Editores.

LeCLerC, Annie (1977). Palabra de mujer, Buenos Aires: Ediciones Megapolis.

MILL, Stuart and Harriet TAYLOR MILL (1970). Essays on Sex Equality, Chicago: University of Chicago Press.

$\mathrm{RICH}$, Ann (1980). «Compulsory Heterosexuality and Lesbian Existence» en Signs: Journal of Women in Culture and Society, vol. 5, n. 4 Summer, pp. 631-660.

SanAHUJAYII, M.G Encarna, Teresa SANZ coll y Rosa SEGarRa martí (1994). 'Luce Irigaray. Entrevista', en DUODA Revista de Estudis Feministes, núm 7, pp. 177-185. En línea: intercambia.educalab.es/wp-content/uploads/2015/04/Irigaray-entrevistaDuoda.pdf

SInger, Irvin (2009). Philosophy of Love. A Partial Summing-up, Cambridge (MA): The MIT Press.

StOller, Silvia (2018). «Silent love. On Irigaray's suggestion of cultivating sexual difference» en García-Andrade, Adriana, Lena Gunnarsson and Anna JónAsdóttir (2018). Feminism and the Power of Love. Interdisciplinary Interventions, London: Routledge.

TAYLOR, Charles (2016). The Language Animal. The Full Shape of the Human Linguistic Capacity, Cambridge: Harvard University Press.

Weedon, Chris (1987). Feminist Practice and Poststructuralist Theory, Oxford: Blackwell.

WheleHAN, Imelda (1995). Modern Feminist Thought. From the Second wave to 'Post-feminism', Edinburgh: Edinburgh University Press.

WHITFORD, Margaret (1991). «Maternal genealogy and the symbolic» en WHITFORD, Margaret (1991). Luce Irigaray. Philosophy in the Feminine, London: Routledge.

WitTIG, Monique (1992). The Straight Mind and Other Essays, Boston: Beacon Press.

WolstoneCRAft, Mary (1996). A Vindication of the Rights of Woman, Seattle: AmazonClassics.

WRIGHT, Elisabeth (ed) (1992). Feminism and Psycoanalysis. A Critical Dictionary, Oxford: Blackwell Publisher. 